\title{
A Novel Dual-Frequency Deep Brain Stimulation Paradigm for Parkinson's Disease
}

\author{
Jessica A. Karl · Bichun Ouyang · Leo Verhagen Metman
}

Received: April 15, 2019 / Published online: June 26, 2019

(C) The Author(s) 2019

\section{ABSTRACT}

Introduction: Deep brain stimulation (DBS) of the subthalamic nucleus (STN) using high-frequency (130-185 Hz) stimulation (HFS) is more effective for appendicular than for axial symptoms. Low-frequency stimulation (LFS) of the STN may reduce gait/balance and speech impairment but can result in worsened appendicular symptoms, limiting its clinical usefulness. A novel dual-frequency paradigm (interleave-interlink, IL-IL) was created in order to reduce gait/balance and speech impairment while maintaining appendicular symptom control in Parkinson's disease (PD) patients chronically stimulated with DBS.

Methods: Two overlapping LFS programs are applied to each DBS lead, with the overlapping area focused around the optimal electrode contact. As a result, this area receives HFS, controlling appendicular symptoms. The nonoverlapping area receives LFS, potentially reducing gait/balance and speech impairment. Patients were separated into three categories

Enhanced digital features To view enhanced digital features for this article go to https://doi.org/10.6084/ m9.figshare.8203493.

J. A. Karl $(\varangle) \cdot$ B. Ouyang · L. Verhagen Metman Movement Disorder Section of Neurological Sciences, Rush University Medical Center, 1725 W. Harrison Street, Suite 755, Chicago, IL 60612, USA e-mail: jessica_karl@rush.edu based on their chief complaint(s): gait/balance impairment, speech impairment, and/or incomplete PD symptom control. The ClinicalGlobal Impression of Change scale (CGI-C) was completed retrospectively based on patient/caregiver feedback in patients who remained on IL-IL (at 3 months and at the last follow-up).

Results: Seventy-six patients were switched from optimized HFS to IL-IL. Fifty-five (72\%) patients remained on IL-IL after $22 \pm 8.7$ months. The median (range) CGI-C for gait was $2(1-5)$ at 3 months and 3 (1-4) at last follow-up, for dysarthria it was 4 (1-4) at 3 months and 4 (1-5) at last follow-up, and for PD motor it was $2(1-3)$ at 3 months and 2 (1-3) at last follow-up.

Conclusion: A substantial number of patients remained on IL-IL because of subjective improvements in gait/balance, speech, or PD symptoms. A prospective, double-blind, crossover study with objective/quantitative outcome measures is underway.

Keywords: Deep brain stimulation; Freezing of gait; Interleaving stimulation; Low-frequency stimulation; Parkinson's disease

\section{INTRODUCTION}

Subthalamic nucleus (STN) deep brain stimulation (DBS) is an established therapy for patients 
with advanced Parkinson's disease (PD). Conventional high-frequency $(130-185 \mathrm{~Hz})$ stimulation (HFS) of the STN offers a long-term benefit for appendicular symptoms, but axial symptoms associated with advancing disease are resistant to or worsened by HFS [1-3]. Several groups have evaluated the effect of lowfrequency $(60-90 \mathrm{~Hz})$ stimulation (LFS) of the STN on freezing of gait, balance impairment, and speech. The results have been variable, but one of the largest drawbacks is the inability to maintain appendicular symptom control, limiting its clinical usefulness $[4,5]$. Interleaving stimulation consists of the rapid and alternate activation of two independent programs on each lead that can have differing amplitudes and pulse widths but are limited to the same frequency. It has been used to reduce stimulation-induced side effects but, because of higher battery drain, it is only recommended when conventional stimulation paradigms fail to achieve a desirable clinical benefit [6, 7].

In the current study, we combined the strengths of low-frequency and interleaving stimulation to create a novel dual-frequency paradigm named interleave-interlink (IL-IL) in order to reduce axial symptoms while preserving appendicular symptom control.

\section{METHODS}

The authors confirm that the work presented in this manuscript is consistent with the Journal of Neurology and Therapy position on ethical publication. The Rush University Medical Center Institutional Review Board (IRB) approved this study (ID \#19012501-IRB01). The study conformed with the Declaration of Helsinki as revised in 2013 concerning human rights. Informed patient consent was not necessary for this work.

\section{Dual Frequency Paradigm (IL-IL) Development}

The IL-IL paradigm is aimed at reducing treatment-resistant gait, balance, and speech problems while preserving appendicular symptom control. We developed IL-IL using interleaving in a way that two overlapping LFS programs are applied to each DBS lead with the overlapping area focused around the optimal electrode contact. The overlapping area receives HFS, providing appendicular symptom control, whereas the non-overlapping areas receive LFS to reduce freezing of gait, balance impairment, and speech impairment.

IL-IL is programmed based on the patients' optimized conventional HFS settings. If the HFS setting lacks two active cathodes, a monopolar survey is completed and the two best electrode contacts are selected. Two overlapping bipolar interleaving programs are then activated (i.e., program 1: $0-2+$, program 2: $1-3+$ ). The pulse width is programmed the same as the HFS setting, and the frequency is reduced by approximately 50\% (i.e., HFS: 185 Hz, IL-IL: 90 Hz). The stimulation amplitude is empirically determined by the programmer based on PD symptom control. If symptoms are not adequately controlled with two bipolar interleaving programs, despite amplitude adjustments, one monopolar and one bipolar or two monopolar configurations are applied (i.e., program 1: 0-2+, program 2: 1-C+ or program 1: $0-C+$, program 2: $1-\mathrm{C}+$ ). Illustrations of a DBS lead without stimulation (a), conventional HFS (b), LFS (c), and IL-IL (d) are depicted in Fig. 1.

\section{Patients}

PD patients chronically stimulated (average 6.9 years) with bilateral Medtronic STN DBS at

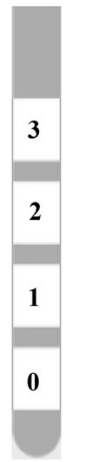

A

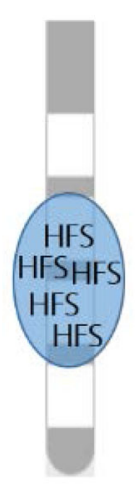

B

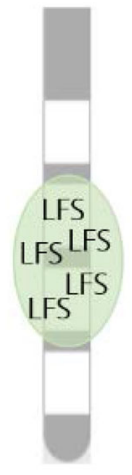

C

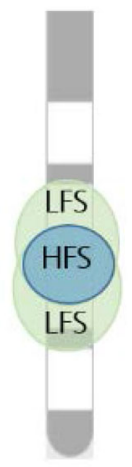

D
Fig. 1 DBS lead (a), conventional HFS (b), LFS (c), and IL-IL (d) 
Rush University Medical Center and programmed with the IL-IL programming paradigm between 2011 and 2017 were identified. All patients programmed with IL-IL had gait and balance impairment, dysarthria, and/or incomplete appendicular symptom control despite optimized conventional HFS settings.

\section{Study Design}

A retrospective chart review was completed in all patients who remained on IL-IL. Patients were separated into three categories based on their chief complaint(s) prior to stimulation adjustment: gait and balance impairment, speech impairment, and/or incomplete PD motor control, with some patients belonging to more than one category. Baseline was determined as the date the IL-IL programming change occurred. The primary outcome measure was the Clinical Global Impression of Change (CGI-C) scale [8]. The anchors are: 1-very much improved, 2-much improved, 3-minimally improved, 4-no change, 5-minimally worse, 6-much worse, 7-very much worse. An unblinded rater determined symptom severity at 3 months and at the last follow-up, comparing each to the baseline. The scores were rated based on patient and caregiver history and the clinician's physical examination as documented in the medical record. The levodopa equivalent daily dose (LEDD) was calculated for all patients at baseline and at last follow-up.

\section{Statistical Analysis}

Descriptive analysis (mean, median, standard deviation, range) was performed as appropriate. In those remaining on IL-IL, a comparison of CGI-C scores at 3 months after stimulation change with those at last follow-up was completed to determine the long-term effect using the Wilcoxon signed rank test. In all patients, comparison of LEDD at baseline to that at last follow-up was completed using the paired $t$ test. The two-sample $t$ test was used to compare LEDD between those who remained on IL-IL and those who returned to their conventional
HFS setting. Statistical significance was set at $p<0.05$.

\section{RESULTS}

\section{Participants}

Seventy-six patients (47 male, 29 female) with IL-IL were identified. Demographic data are described in Table 1 . The majority were stimulated with two bipolar (i.e., $0-2+, 1-3+$ ) overlapping programs $(n=49)$. The remaining were either stimulated with one bipolar and monopolar (i.e., $0-2+, 1-\mathrm{C}+)$ program $(n=24)$ or two monopolar (i.e., $0-\mathrm{C}+, 1-\mathrm{C}+$ ) programs $(n=3)$. Seventy-two percent $(n=55)$ of patients remained on IL-IL after an average of $22 \pm 8.7$ months. Twelve percent $(n=9)$ of patients returned to their conventional HFS setting, with the average time spent on IL-IL being 5 days (range 1-22 days). The main reason for abandoning the IL-IL paradigm was incomplete appendicular symptom control. At the last follow-up, $16 \%(n=12)$ of patients had not returned for follow-up.

\section{Rating scale and LEDD}

The patients who remained on IL-IL $(n=55)$ were separated into three categories based on their chief complaints(s): gait and balance impairment $(n=48)$, speech impairment $(n=17)$, and/or incomplete PD motor control $(n=14)$, with some patients belonging to more than one group. The median (range) CGI-C for gait and balance impairment was 2-much improved (1-5) at 3 months and 3-minimally improved (1-4) at the last follow-up. For speech

Table 1 Baseline demographics $(n=76)$

\begin{tabular}{ll}
\hline Baseline characteristic & Mean (SD) \\
\hline Age (years) & $65.9(7.0)$ \\
Disease duration (years) & $18.3(6.5)$ \\
Years since DBS surgery & $6.9(4.3)$ \\
LEDD & $657.5(491.80)$ \\
\hline
\end{tabular}


impairment the median (range) CGI-C was 4-no change (1-4) at 3 months and 4-no change (1-5) at last follow-up, and for incomplete PD motor control 2-much improved (1-3) at 3 months and 2-much improved (1-3) at the last follow-up. There was no significant difference between the CGI-C scores at 3 months and at the last follow-up for gait and balance impairment $(z=-0.406, p=0.685)$, speech impairment $(z=-1.000, p=0.317)$, and incomplete PD motor control $(z=-1.000, p=0.317)$ (Fig. 2). There was no difference in the mean change (SD) in LEDD between those who remained on the novel IL-IL paradigm $(55.4 \pm 290.4)$ and those who returned to their conventional HFS setting $(26.4 \pm 146.8)$, $p=0.65$ (Table 2).

\section{DISCUSSION}

\section{Overall Outcome}

This is the first study of combined low- and high-frequency stimulation in a novel dual-
Table 2 Mean (SD) change in LEDD from before IL-IL was implemented to the last follow-up ( $22 \pm 8.7$ months) for all patients

\begin{tabular}{lrcc}
\hline & \multicolumn{1}{l}{ IL-IL } & $\begin{array}{l}\text { Conventional } \\
\text { HFS } \\
\text { Mean (SD) }\end{array}$ & $\boldsymbol{p}$ value \\
\hline $\begin{array}{l}\text { LEDD } \\
\text { before }\end{array}$ & $634(417)$ & $790(659)$ & 0.51 \\
$\begin{array}{l}\text { LEDD after } \\
\text { Change }\end{array}$ & $-578(389)$ & $763(636)$ & 0.42 \\
\hline
\end{tabular}

frequency paradigm (IL-IL) for conventional HFS-refractory axial symptoms. In this large group of patients, the majority chose to remain on IL-IL because of reductions in gait, balance, and speech impairment. Appendicular symptom control was maintained in the group that stayed on IL-IL and some patients reported an improvement compared to HFS. This is important to note given that unsatisfactory

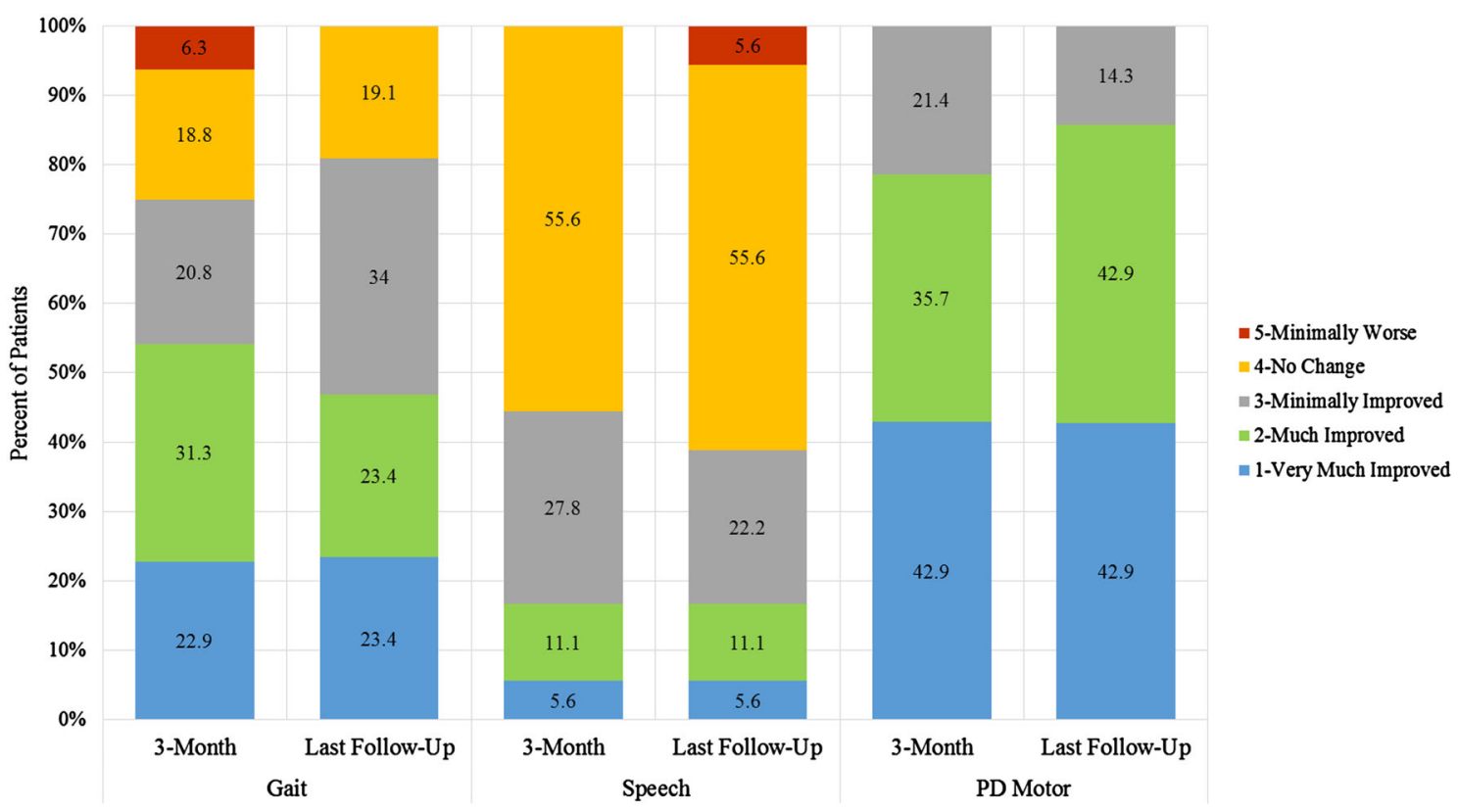

Fig. 2 Distribution of CGI-C scores at 3 months and at last follow-up among patients who remained on IL-IL. Patients were grouped into three categories based on their chief complaint(s): gait and balance impairment (gait),

speech impairment (speech), and/or incomplete PD motor control (PD motor); some patients belonged to more than one group 
appendicular symptom control is one of the main limitations of LFS [4].

\section{Long-Term Effect}

The reported benefits were maintained for a long period of time ( $22 \pm 8.7$ months). This is in contrast to reports on the effect of LFS, where, after an initial improvement, benefits waned over time, and LFS had to be aborted [9-13]. It is important to note that the LEDD remained unchanged after IL-IL was implemented, suggesting that the improvements observed were not the result of medication adjustment.

\section{Battery}

Interleaving stimulation using high-frequency $(125 \mathrm{~Hz})$ is a recommended strategy for improving symptoms and reducing side effects in patients with a suboptimal response to conventional troubleshooting strategies $[6,7]$. Because of higher battery drain, it is typically not used until other programming strategies have been exhausted [14]. Because lower frequencies $(60-90 \mathrm{~Hz})$ are used in the IL-IL paradigm, battery depletion is less of a limitation. In some instances, the use of IL-IL may save energy. For example, a frequency of $60 \mathrm{~Hz}$ in the IL-IL paradigm would cause less of a battery drain than a conventional HFS frequency of 130-185 Hz.

\section{Premature Return}

A minority of the patients were not satisfied with IL-IL and returned to their conventional HFS settings, mainly because of incomplete appendicular symptom control. This may have been premature, as it took several clinic visits to optimize the IL-IL stimulation setting for many of the patients. It has been suggested that 'total electrical energy delivered' calculations should be completed when evaluating a single parameter (i.e., frequency) [15]. This was not done when converting stimulation settings from conventional HFS to IL-IL because more than one variable (electrode configuration and frequency) was being adjusted.

\section{Mechanism of Action}

The mechanism of action of IL-IL remains to be determined. One explanation is that less-frequent delivery of stimulation pulses to structures adjacent to the STN (i.e., internal capsule) reduces side effects such as gait, balance impairment, and dysarthria. Alternatively, the application of LFS may be essential because of connections between the STN and pedunculopontine nucleus, a locomotor center that has been shown to respond to lower frequencies [16-18]. Another reason may be simultaneous stimulation of the pars reticulata in the substantia nigra $(\mathrm{SNr})$ and the STN. Valledeoriola et al. found a reduction in gait impairment with both LFS $(63 \mathrm{~Hz})$ delivery to the $\mathrm{SNr}$ and STN [19]. In the same vein, Khoo et al. found that delivering LFS to the ventral STN was favorable for reducing axial symptoms, and recommended optimizing active contacts with respect to frequency [10]. We are currently investigating whether the LFS field is located in the $\mathrm{SNr}$ or within specific regions of the STN using the Suretune $^{\mathrm{TM}}$ [3] imaging software system.

\section{Study Limitations}

There are limitations of our study that are worth noting. The retrospective nature of the data collection carried out by an unblinded rater has obvious limitations. In addition, no quantitative measures were used to capture symptom severity. However, given the increasing significance and relevance of patient-reported outcomes, we used the CGI-C scale, which is based on the patients' and caregivers' perceptions of DBS programming adjustments.

\section{CONCLUSION}

A treatment that addresses axial symptoms in PD remains critically needed. This is the first study to evaluate the effect of combined lowand high-frequency stimulation on axial 
symptoms. In this retrospective study, a significant number of patients chose to remain on IL-IL after an average of 22 months because of subjective improvements in gait and balance, speech, and/or cardinal PD symptoms. This suggests that the proposed dual-frequency paradigm (IL-IL) may be useful in selected patients with continued axial symptom impairment that cannot otherwise be controlled with conventional stimulation programming techniques. We have now started a prospective randomized, double-blind, crossover trial to confirm these preliminary findings.

\section{ACKNOWLEDGEMENTS}

The Rush Parkinson's Disease and Movement Disorder Program is a designated Parkinson's Foundation Center of Excellence.

Funding. This research did not receive any specific grant from funding agencies in the public, commercial, or not-for-profit sectors.

Authorship. All named authors meet the International Committee of Medical Journal Editors criteria for authorship for this article, take responsibility for the integrity of the work as a whole, and have given their approval for this version to be published.

Author Contributions. JAK: research project: conception, organization and execution; statistical analysis: design, execution, review and critique; manuscript preparation: writing of first draft, review and critique. BO: statistical analysis: design, execution, review and critique. LVM: research project: conception; statistical analysis: review and critique; manuscript preparation: review and critique.

Disclosures. Jessica A. Karl, Bichun Ouyang, and Leo Verhagen Metman have nothing to declare. Leo Verhagen Metman is a member of the journal's Editorial Board.

Compliance with Ethics Guidelines. The Rush University Medical Center Institutional Review Board (IRB) approved this study (ID
\#19012501-IRB01). The study conforms with the 1964 Declaration of Helsinki of 1964, as revised in 2013, concerning human rights. Informed patient consent was not necessary for this work.

Data Availability. The datasets generated and/or analyzed during the current study are not publicly available for patient confidentiality reasons, but are available from the corresponding author on reasonable request.

Open Access. This article is distributed under the terms of the Creative Commons Attribution-NonCommercial 4.0 International License (http://creativecommons.org/licenses/ by-nc/4.0/), which permits any noncommercial use, distribution, and reproduction in any medium, provided you give appropriate credit to the original author(s) and the source, provide a link to the Creative Commons license, and indicate if changes were made.

\section{REFERENCES}

1. Castrioto A, Lozano AM, Poon YY, Lang AE, Fallis M, Moro E. Ten-year outcome of subthalamic stimulation in Parkinson disease: a blinded evaluation. Arch Neurol. 2011;68(12):1550-6.

2. Fasano A, Romito LM, Daniele A, et al. Motor and cognitive outcome in patients with Parkinson's disease 8 years after subthalamic implants. Brain. 2010;133(9):2664-76.

3. Gervais-Bernard H, Xie-Brustolin J, Mertens P, et al. Bilateral subthalamic nucleus stimulation in advanced Parkinson's disease: five year follow-up. J Neurol. 2009;256(2):225-33.

4. Moreau C, Defebvre L, Destee A, et al. STN-DBS frequency effects on freezing of gait in advanced Parkinson disease. Neurology. 2008;71(2):80-4.

5. Phibbs FT, Arbogast PG, Davis TL. $60-\mathrm{Hz}$ frequency effect on gait in Parkinson's disease with subthalamic nucleus deep brain stimulation. Neuromodulation. 2014;17(8):717-20.

6. Zhang S, Zhou P, Jiang S, Wang W, Li P. Interleaving subthalamic nucleus deep brain stimulation to avoid side effects while achieving satisfactory motor benefits in Parkinson disease: a report of 12 cases. Medicine (Baltimore). 2016;95(49):e5575. 
7. Miocinovic S, Khemani P, Whiddon R, et al. Outcomes, management, and potential mechanisms of interleaving deep brain stimulation settings. Parkinsonism Relat Disord. 2014;20(12):1434-7.

8. Busner J, Targum SD. The Clinical Global Impressions Scale: applying a research tool in clinical practice. Psychiatry (Edgmont). 2007;4(7):28-37.

9. Sidiropoulos C, Walsh R, Meaney C, Poon YY, Fallis M, Moro E. Low-frequency subthalamic nucleus deep brain stimulation for axial symptoms in advanced Parkinson's disease. J Neurol. 2013;260(9):2306-11.

10. Khoo HM, Kishima H, Hosomi K, et al. Low-frequency subthalamic nucleus stimulation in Parkinson's disease: a randomized clinical trial. Mov Disord. 2014;29(2):270-4.

11. Vallabhajosula S, Haq IU, Hwynn N, et al. Lowfrequency versus high-frequency subthalamic nucleus deep brain stimulation on postural control and gait in Parkinson's disease: a quantitative study. Brain Stimul. 2015;8(1):64-75.

12. Ricchi V, Zibetti M, Angrisano S, et al. Transient effects of $80 \mathrm{~Hz}$ stimulation on gait in STN DBS treated PD patients: a 15 months follow-up study. Brain Stimul. 2012;5(3):388-92.

13. Brozova H, Barnaure I, Alterman RL, Tagliati M. STN-DBS frequency effects on freezing of gait in advanced Parkinson disease. Neurology. 2009;72(8):770-1.
14. Ramirez-Zamora A, Kahn M, Campbell J, DeLaCruz $P$, Pilitsis JG. Interleaved programming of subthalamic deep brain stimulation to avoid adverse effects and preserve motor benefit in Parkinson's disease. J Neurol. 2015;262(3):578-84.

15. di Biase L, Fasano A. Low-frequency deep brain stimulation for Parkinson's disease: great expectation or false hope? Mov Disord. 2016;31(7):962-7.

16. Mazzone P, Paoloni M, Mangone $\mathrm{M}$, et al. Unilateral deep brain stimulation of the pedunculopontine tegmental nucleus in idiopathic Parkinson's disease: effects on gait initiation and performance. Gait Posture. 2014;40(3):357-62.

17. Moro E, Hamani C, Poon YY, et al. Unilateral pedunculopontine stimulation improves falls in Parkinson's disease. Brain. 2010;133(Pt 1):215-24.

18. Welter ML, Demain A, Ewenczyk C, et al. PPNa-DBS for gait and balance disorders in Parkinson's disease: a double-blind, randomised study. J Neurol. 2015;262(6):1515-25.

19. Valldeoriola F, Munoz E, Rumia J, et al. Simultaneous low-frequency deep brain stimulation of the substantia nigra pars reticulata and high-frequency stimulation of the subthalamic nucleus to treat levodopa unresponsive freezing of gait in Parkinson's disease: a pilot study. Parkinsonism Relat Disord. 2019;60:153-7. 